\title{
Issues on Potential Growth Measurement and Comparison: How Structural Is the Production Function Approach?
}

\author{
Christophe Cahn and Arthur Saint-Guilhem
}

\begin{abstract}
This article aims to better understand the factors driving fluctuations in potential output measured by the production function approach (PFA.) To do so, the authors integrate a production function definition of potential output into a large-scale dynamic stochastic general equilibrium (DSGE) model in a fully consistent manner and give two estimated versions based on U.S. and euro-area data. The main contribution of this article is to provide a quantitative and comparative assessment of two approaches to potential output measurement, namely DSGE and PFA, in an integrated framework. The authors find that medium-term fluctuations in potential output measured by the PFA are likely to result from a large variety of shocks, real or nominal. These results suggest that international comparisons of potential growth using the PFA could lead to overstating the role of structural factors in explaining cross-country differences in potential output, while neglecting the fact that different economies are exposed to different shocks over time. (JEL C51, E32, O11, O47)
\end{abstract}

Federal Reserve Bank of St. Louis Review, July/August 2009, 91(4), pp. 221-40.

nternational comparisons of potential output growth have received renewed interest in recent years. Lower economic performance in Europe compared with the United States over the past 15 years has generated several publications whose aim is to explain the sources of divergence in economic performance and which question how to enhance economic growth in Europe. In line with the recommendations of the Lisbon strategy, one general conclusion is that structural reforms should help to sustain more vigorous growth in Europe and enable European economies to catch up to the United States. Such reforms include labor and product market liberalization, public policies to encourage innovation, and so forth. Examples can be found in most recent International Monetary Fund (IMF) or
Organisation for Economic Co-operation and Development (OECD) country reports on European economies. For instance, the 2007 IMF Article IV Staff Report for France (IMF, 2007) typically incorporates, among others, the important conclusion that "economic policy needs to address the root cause of France's growth deficit: the weakness of its supply potential." Against this background, it is important to have a clear view on how potential output is measured and what interpretation can be made of cross-country differences in potential output growth.

Among the different methods of measurement of potential output, the production function approach (PFA) is probably the most widely used. With this approach, output growth is expressed as a sum of the growth of factor inputs (i.e., capital

Christophe Cahn is a doctoral candidate at the Paris School of Economics and an economist with the Banque de France. Arthur Saint-Guilhem is an economist with the European Central Bank. The authors thank Jon Faust for his helpful comments, as well as Richard Anderson and all the participants at the conference.

(C) 2009, The Federal Reserve Bank of St. Louis. The views expressed in this article are those of the author(s) and do not necessarily reflect the views of the Federal Reserve System, the Board of Governors, the regional Federal Reserve Banks, the European Central Bank, the Banque de France, or the Paris School of Economics. Articles may be reprinted, reproduced, published, distributed, displayed, and transmitted in their entirety if copyright notice, author name(s), and full citation are included. Abstracts, synopses, and other derivative works may be made only with prior written permission of the Federal Reserve Bank of St. Louis. 
services and labor input) and a residual (i.e., total factor productivity [TFP] growth). Additional assumptions are made on the potential level of the factors of production. For instance, potential labor input would be calculated by smoothing some variables (such as total population and the participation rate) and by approximating the medium-term equilibrium unemployment rate with the non-accelerating inflation rate of unemployment. The major advantage of the PFA, compared with statistical aggregate methods, is that it provides an economic interpretation of the different factors that drive growth in potential output. This is especially useful in the context of international comparisons. Moreover, conducting additional econometric analysis allows use of the PFA as a framework to capture the impact on potential growth of major changes, such as the pickup in productivity growth that started in the second half of the 1990s in the United States.

However, this approach raises some difficulties. Estimates of the components are bounded by a large degree of uncertainty because analysis results are highly dependent on the choice of modeling of the different components-for instance, how trend growth of TFP is estimated. Another difficulty derives from possible misleading interpretations of potential output as measured by the PFA. First, in the context of international comparisons, cross-country differences in PFA potential output are often given a structural interpretationsay, as being caused by different degrees of rigidities in the labor or good markets, whereas these differences in potential output measures could reflect only the lasting effects of temporary shocks to the economy. This issue is of particular importance because it casts doubt on the ability of the PFA to give a satisfactory picture of the structural components of economic growth. Second, the PFA leaves unidentified the various shocks (supply, demand, monetary shocks, and so on) that are likely to affect potential output in the medium term. This raises some concern about the measurement of output gaps. Indeed, it is not entirely certain that fluctuations in the output gap measured by the PFA reflect only inflation-related shocks. Therefore, the PFA might lead to biased output gap measures that could make them unreliable for the assessment of monetary policy conditions.
An alternative approach to the definition and measurement of potential output can be found in New Keynesian dynamic stochastic general equilibrium (DSGE) models. The recent literature on DSGE models has shown significant progress in developing models that can be applied to the data. Indeed, recent research has shown that estimated DSGE models are able to match the data for key macroeconomic variables and reduced-form vector autoregressions (Smets and Wouters, 2007). In these models, "potential output" is generally defined as the level of output that would prevail in an economy with fully flexible prices and wages. According to the DSGE definition, potential output is therefore the level of output at which prices tend to stabilize. However, the properties of potential output and output gap fluctuations derived from DSGE models can be quite different from the ones derived from the PFA (e.g., Neiss and Nelson, 2005; and Edge, Kiley, and Laforte, 2007). For example, the DSGE measure of potential output can undergo relatively larger fluctuations than potential output derived from the PFA. Similarly, the output gap in DSGE models tends to be less variable than with the PFA measures. One caveat of these papers, however, is that they compare ad hoc PFA measures of potential output with DSGE measures-comparisons that would be enhanced if the PFA measure of potential output were consistent with the model. In this respect, one of the main contributions of our paper is to incorporate the PFA measure of potential output into a DSGE framework in a fully consistent manner. As shown later, adopting such a method reveals that different types of shocks are likely to cause potential output measured by the PFA to fluctuate.

Our goals are twofold: (i) better understanding of the factors driving medium-term fluctuations in the PFA potential output and (ii) providing a quantitative comparison of the PFA versus DSGE measure of potential output. To do so, we build a large-scale DSGE model, calibrate two versions of the model using U.S. and euro-area data, and then integrate into this framework a PFA definition of potential output that is fully consistent with the model. Our PFA is based on previous 
work (Cahn and Saint-Guilhem, 2009), where output of the economy is described as a Cobb-Douglas function. In this respect, the main contribution of this paper is to provide a quantitative comparison of these two measures of potential outputthe PFA versus the DSGE-in a fully integrated conceptual framework-namely, an economy modeled as a large-scale DSGE model with structural parameters calibrated on U.S. and euro-area data and with an alternative PFA measure of potential output.

A second contribution of this paper is to assess the validity of the structural interpretation of crosscountry comparisons of potential output measures given by the PFA. In general, as described previously, potential output estimates based on the PFA suggest significant differences across countries with regard to the sources of potential growth. However, whether these differences can be attributed to structural factors, such as differences in labor market or product market institutions, remains uncertain. Nothing in the PFA guarantees that this is the case. Our present DSGE framework enables us to tackle the issue, given that in such a framework structural differences across two economies translate into differences of magnitude across the various parameters of the model. We can therefore quantify the role of shocks versus the role of structural factors in explaining crosscountry differences in potential output measured by the PFA by simulating various counterfactual scenarios for the two model economies.

Our main results first confirm that the PFA and the DSGE definitions of potential output are two different concepts. We find that in an economy modeled with a DSGE framework, medium-term fluctuations in potential output measured by the PFA result from a variety of shocks, such as productivity or monetary shocks. We also find that differences in potential output between two such model economies as measured by the PFA can be attributed not only to structural parameters of the model but also to the role of some transitory shocks, real or nominal, affecting the economies. If we transpose these results into the empirical field, we see two results: (i) PFA measures of potential output also reflect the historical pattern of shocks that affect a given economy, and (ii) international comparisons of potential output using the PFA could lead to overestimating the role of structural factors in explaining crosscountry differences in potential output, while neglecting the role of "luck," namely, the fact that different economies are exposed to different histories of stochastic events on which structural policies could not act.

The remainder of this paper is organized as follows. In the next section, we sketch the theoretical specification of our DSGE model. The following section describes how we incorporate and implement into this framework the PFA measure of potential output. We then present and discuss the results of the simulations performed with regard to the decomposition of potential output dynamics into the contributions of the various shocks included in the model. Our summary and conclusion then follow.

\section{A BENCHMARK DSGE MODEL FOR THE UNITED STATES AND THE EURO AREA}

In this section, we provide details on the main optimizing behaviors of economic agentshouseholds, firms, and the fiscal and monetary authorities-that lead to building the equations of our benchmark DSGE model, which is largely taken from Smets and Wouters (2007). ${ }^{1}$

\section{The Representative Household}

We consider an economy populated by a representative household with access to financial and physical capital markets so that trading in bonds, investment goods, and state-contingent securities can occur. Household wealth is given by gains from government bonds in nominal per capita terms, $B_{t-1}$, held at the beginning of period $t$. Labor income comes from the nominal wage rate, $W_{t}^{h}$, and homogeneous labor, $l_{t}^{h}$, pooled by a set of labor unions, $u \in[0,1]$. Households receive nominal dividends, $\Pi_{t}^{f}$ and $\Pi_{t}^{u}$, from intermediate producers and labor unions, respectively. Capital

1 Detailed equations are given in a technical appendix not included here. The appendix and Dynare codes are available on request from the authors. 
services incomes are $r_{t}^{K} \tilde{K}_{t}$, where $r^{K}$ is the real rental price of capital service, $\tilde{K}$.

These revenues are used to pay for consumption, $P_{t} C_{t}$, and investment, $P_{t} I_{t}$, goods, and for lump-sum taxes expressed in the output price, $P_{t} T_{t}$. Moreover, the representative household buys discounted government bonds due at the end of period $t, B_{t} /\left(\varepsilon_{t}^{b} R_{t}\right)$, where $\varepsilon_{t}^{b}$ is a risk premium shock. Hence, the budget constraint of such a household is given by the following:

$$
\begin{aligned}
& P_{t} C_{t}+P_{t} I_{t}+P_{t} T_{t}+B_{t} /\left(\varepsilon_{t}^{b} R_{t}\right) \\
& \leq W_{t}^{h} l_{t}^{h}+P_{t} r_{t}^{K} \tilde{K}_{t}+\Pi_{t}^{f}+\Pi_{t}^{u}+B_{t-1},
\end{aligned}
$$

which expressed in real terms becomes

$$
\begin{aligned}
& C_{t}+I_{t}+T_{t}+B_{t} /\left(\varepsilon_{t}^{b} R_{t} P_{t}\right) \leq w_{t}^{h} l_{t}^{h}+ \\
& r_{t}^{K} \tilde{K}_{t}+\left(\Pi_{t}^{f}+\Pi_{t}^{u}\right) / P_{t}+B_{t-1} / P_{t},
\end{aligned}
$$

where $w_{t}^{h}=W_{t}^{h} / P_{t}$ is the real wage received by the household.

Capital services come from the combination of physical capital, $K_{t}$, adjusted by capacity utilization, $z_{t}$, such that $\tilde{K}_{t}=z_{t} K_{t-1}$. Physical capital accumulation implies adjustment cost on investment change, $S(\cdot)$, and the time-varying depreciation process, $\delta(\cdot)$, according to

$$
K_{t}=\left[1-\delta\left(z_{t}\right)\right] K_{t-1}+\left(1-S\left(\varepsilon_{t}^{i} \frac{I_{t}}{I_{t-1}}\right)\right) I_{t},
$$

where $\varepsilon_{t}^{i}$ is a shock that deforms the adjustment cost function. ${ }^{2}$

We define the intertemporal utility function as follows:

$$
U_{t}=E_{t}\left\{\begin{array}{l}
\sum_{j=0}^{+\infty} \beta^{j} \frac{\left(C_{t+j}-\Theta_{t+j}\right)^{1-\sigma_{c}}}{1-\sigma_{c}} \times \\
\exp \left[\eta \varepsilon_{t}^{l} \frac{\sigma_{C}-1}{1+\sigma_{l}}\left(l_{t+j}^{h}\right)^{1+\sigma_{l}}\right]
\end{array}\right\},
$$

where $\sigma_{C}$ is the intertemporal substitution parameter of consumption, $\sigma_{l}$ the intertemporal substitution elasticity of labor, $\eta$ a scale parameter, and

2 We depart from the Smets and Wouters (2007) model by substituting the initial cost function on change in capital with a time-varying depreciation rate. $\varepsilon_{t}^{l}$ a labor supply shock. External habits are given by $\Theta_{t}=\theta C_{t-1}, 0<\theta<1$.

The representative household's problem consists of maximizing its intertemporal utility subject to its budget constraint and capital accumulation by choosing the path of $C_{t}, I_{t}, B_{t}, z_{t}, K_{t}$, and $l_{t}^{h}$.

\section{Supply Side}

We consider a continuum of intermediate goods producers, $f \in[0,1]$. Each intermediate firm produces a differentiated good used in the production of a final good. Following Kimball (1995), the aggregation function is implicitly given by the following condition:

$$
\int_{0}^{1} G^{Y}\left(\varepsilon_{t}^{y} \frac{y_{t}(f)}{Y_{t}}\right) d f=1,
$$

where $G^{Y}(\cdot)$ is an increasing, concave function and verifies $G(1)=1$ and $\varepsilon_{t}^{y}$ is a shock that distorts the aggregator function. The representative firm in the final good sector maximizes its profit given the prices of intermediate goods, $P_{t}(f)$, and the price of the final good, $P_{t}$.

We assume the following technology in the intermediate producer sector:

$$
y_{t}(f)=\varepsilon_{t}^{a} \tilde{K}_{t}(f)^{\alpha}\left((1+g)^{t} L_{t}(f)\right)^{1-\alpha},
$$

where $\varepsilon_{t}^{a}$ is a productivity shock,

$$
\begin{aligned}
& \ln \left(\varepsilon_{t}^{a}\right)=\left(1-\rho_{a}\right) \ln \left(\bar{\varepsilon}^{a}\right)+\rho_{a} \varepsilon_{t-1}^{a}+v_{t}^{a}, \\
& v_{t}^{a} \sim N\left(0, \sigma_{a}\right),
\end{aligned}
$$

and $g$ is the growth rate of a deterministic, Harrodneutral technological trend. Assuming that the input markets are perfectly competitive, a firm $f \in[0,1]$ chooses an input mix, $\left(\tilde{K}_{t}(f), L_{t}(f)\right)$, by solving the following program:

$$
\begin{aligned}
\min _{\left\{\tilde{K}_{t}(f), L_{t}(f)\right\}} w_{t} L_{t}(f)+r_{t}^{K} \tilde{K}_{t}(f) \\
\text { s.t. } y_{t}(f)=\varepsilon_{t}^{a} \tilde{K}_{t}(f)^{\alpha}\left((1+g)^{t} L_{t}(f)\right)^{1-\alpha},
\end{aligned}
$$

where the real aggregate labor price, $w_{t}$, and rental capital rate, $r_{t}^{K}$, are given.

Firms are not allowed to optimally reset their price at each date. With probability $\xi_{p}>0$, the 
firm, $f$, cannot optimally adjust its price at time $t$; instead, it follows the following rule:

$$
P_{t}(f)=\bar{\pi}_{t}^{1-\gamma_{p}} \pi_{t-1}^{\gamma_{p}} P_{t-1}(f)=\Gamma_{t}^{p} P_{t-1}(f) ;
$$

that is, a nonoptimizing firm sets its price by indexing the current price on a convex combination of past inflation and the inflation target, to be defined subsequently.

The intermediate firm's problem can be written as follows:

$$
\max _{\tilde{P}_{t}(f)} E_{t}\left\{\begin{array}{l}
\sum_{j=0}^{+\infty}\left(\beta \xi_{p}\right)^{j} \frac{\lambda_{t+j}}{\lambda_{t}} \frac{P_{t}}{P_{t+j}} \\
{\left[P_{t+j}(f)-P_{t+j} m c_{t+j}\right] y_{t+j}(f)}
\end{array}\right\}
$$

under conditions

$$
P_{t+j}(f)=\Gamma_{t, j}^{p} \tilde{P}_{t}(f) \text { with } \Gamma_{t, j}^{p} \equiv\left\{\begin{array}{r}
\prod_{s=1}^{j} \Gamma_{t+s}^{p} \text { if } j>0 \\
1 \text { if } j=0
\end{array}\right.
$$

and the relative demand function faced by the intermediate firm.

\section{Wage Setting}

In this economy, the representative household supplies homogeneous labor, $l_{t}^{h}$, to a unitary continuum of intermediate labor unions indexed by $u$. Household and labor unions are price takers with regard to the price, $W_{t}^{h}$, of this type of labor, for which the real counterpart corresponds to the marginal rate of substitution of consumption for leisure. The intermediate labor unions aim at differentiating the household's labor and sell this outcome, $l_{t}(u)$, to a labor agency, setting its price, $W_{t}(u)$, according to a mechanism à la Calvo (1983). Then the labor agency aggregates these differentiated labor services into a labor package, $L_{t}$, and supplies it to productive firms.

Consequently, we assume that the labor agency offers a labor aggregate, $L_{t}$, to intermediate firms, derived from differentiated labor unions, $l_{t}(i)$, according to

$$
\int_{0}^{1} G^{L}\left(\varepsilon_{t}^{W} \frac{l_{t}(i)}{L_{t}}\right) \mathrm{d} i=1
$$

where $G^{L}(\cdot)$ is an increasing, concave function and verifies $G^{L}(1)=1$ and $\varepsilon_{t}^{W}$ a stochastic shock that distorts the aggregator function. Hence, the labor agency maximizes its profit given by

$$
\Pi_{t}=W_{t} L_{t}-\int_{0}^{1} W_{t}(i) I_{t}(i) \mathrm{d} i .
$$

Then, the labor unions set their prices following a Calvo scheme, facing the previous relative demand function and given the wage rate paid to households, $W_{t}^{h}$. More precisely, each labor union seeks to maximize its discounted cash flows by setting the wage rate, $\tilde{W}_{t}(u)$. With probability $\xi_{w}$, the union cannot optimally adjust its wage rate at time $t$; instead, the union adjusts the wage from consumer price inflation according to the following rule:

$$
W_{t}(u)=\bar{\pi}_{t}^{1-\gamma_{w}} \pi_{t-1}^{\gamma_{w}}(1+g) W_{t-1}(u)=\Gamma_{t}^{W} W_{t-1}(u) .
$$

With probability $1-\xi_{w}$, the union is able to choose the optimal wage $\tilde{W}_{t}(u)$. The labor union's problem can be written as follows:

$$
\max _{\tilde{W}(u)} E_{t}\left\{\begin{array}{l}
\sum_{j=0}^{+\infty}\left(\beta \xi_{w}\right)^{j} \frac{\lambda_{t+j}}{\lambda_{t}} \frac{P_{t}}{P_{t+j}} \\
{\left[W_{t+j}(u)-W_{t+j}^{h}\right] l_{t+j}(u)}
\end{array}\right\}
$$

with the following condition:

$$
\begin{aligned}
& W_{t+j}(f)=\Gamma_{t, j}^{w} \tilde{W}_{t}(f) \\
& \text { with } \Gamma_{t, j}^{w} \equiv\left\{\begin{array}{r}
\prod_{s=1}^{j} \Gamma_{t+s}^{w} \text { if } j>0 \\
1 \text { if } j=0
\end{array}\right.
\end{aligned}
$$

and subject to the relative demand function faced by the labor union.

\section{Government, Nominal Distortions, and Aggregation}

We assume that government bonds and transfers evolve according to

$$
P_{t} T_{t}+B_{t} /\left(\varepsilon_{t}^{b} R_{t}\right)=B_{t-1}+P_{t} G_{t},
$$

where $G_{t}$ is an exogenous process such that the ratio $G / Y=\varepsilon_{t}^{g}$ follows an AR(1) process in log. ${ }^{3}$ In addition, the central bank sets the current inter-

3 We use the terms "government shocks" and "external shocks" interchangeably in the following text. 
est rate according to the following Taylor rule in its nonlinear form:

$$
\begin{aligned}
& R_{t}=\bar{R}^{1-\rho_{r}} R_{t-1}^{\rho_{r}}\left[\left(\frac{\pi_{t}}{\bar{\pi}_{t}}\right)^{\phi_{\pi}}\left(\frac{Y_{t}}{Y_{t}^{D S G E}}\right)^{\phi_{y}}\right]^{1-\rho_{r}} \times \\
& \left(\frac{Y_{t}}{Y_{t-1}} \frac{Y_{t-1}^{D S G E}}{Y_{t}^{D S G E}}\right)^{r_{\Delta y}}\left(\frac{\pi_{t}}{\pi_{t-1}}\right)^{r_{\Delta \pi}} \varepsilon_{t}^{m},
\end{aligned}
$$

where $\rho_{r}$ represents the central bank's preference for a smooth interest rate, $\varepsilon_{t}^{m}$ is a monetary shock, $\bar{\pi}_{t}$ is a time-varying inflation target, and $Y_{t}^{D S G E}$ is the output given by a fictional world without nominal rigidities, that is, by setting $\xi_{p}$ and $\xi_{w}$ to zero. Hence, this is a measure of the potential output of such a fictional economy.

Despite the heterogeneity of the wages and prices due to the Calvo scheme, we are able to define aggregates for this economy. In fact, total production, that is, the sum of all productions from intermediate firms, $y_{t}$, is a priori different from the aggregate final product, $Y_{t}$. Consequently, a price distortion, $D_{t}^{p}$, exists such that $y_{t}=Y_{t} D_{t}^{p}$. The same considerations apply as for the labor market. Total work effort provided by the representative household is $l_{t}^{h}$. Hence, a wage dispersion exists such that $l_{t}^{h}=D_{t}^{w} L_{t}{ }^{4}$

We now close the model by deriving the clearing condition on the final product market. First, we need to compute aggregate dividends from intermediate firms:

$$
\Pi_{t}^{f}=\int_{0}^{1}\left[P_{t}(f)-P_{t} m c_{t}\right] y_{t}(f) \mathrm{d} f=P_{t} Y_{t}-P_{t} m c_{t} y_{t}
$$

Aggregate dividends from labor unions are

$$
\Pi_{t}^{u}=\int_{0}^{1}\left[W_{t}(u)-W_{t}^{h}\right] I_{t}(u) \mathrm{d} u=W_{t} L_{t}-W_{t}^{h} I_{t} .
$$

Combining these two equations with the household's and government nominal budget constraints, and using the competitive market condition for production inputs, leads to

$$
C_{t}+I_{t}+G_{t}=Y_{t}
$$

4 In fact, these nominal distortions disappear in a linearized model, as in Smets and Wouters (2007). Nevertheless, we need to deal with these distortions as we plan to simulate the model at the second order.

\section{ESTIMATION AND IMPLEMENTATION OF THE PFA METHOD}

In this section, we first present the estimation of the two versions of the model on U.S. and euroarea data. Then we describe how we integrate a potential output measure based on the PFA into the model in a fully consistent manner.

\section{Functional Forms and Stochastic Structure}

For estimation and simulation, we choose the following functional forms for investment adjustment costs, time-varying depreciation adapted from Greenwood, Hercowitz, and Huffman (1988), and Kimball aggregators that follow the specifications of Dotsey and King (2005):

$$
\begin{aligned}
S(x) & =\frac{(1-x /(1+g))^{2}}{2 \varphi}, \varphi>0 \\
\delta(z)= & \psi_{1}+\psi_{2} \frac{z^{d}}{d} \\
G^{i}(x) & =\frac{1}{\left(1+\omega_{i}\right) \varsigma_{i}}\left[\left(1+\omega_{i}\right) x-\omega_{i}\right]^{\varsigma_{i}} \\
& +\left[1-\frac{1}{\left(1+\omega_{i}\right) \varsigma_{i}}\right], i \in\{Y, L\} .
\end{aligned}
$$

We choose the following stochastic structure for the exogenous processes in this model:

$$
\ln \left(\varepsilon_{t}^{\kappa}\right)=\rho_{\kappa} \ln \left(\varepsilon_{t-1}^{\kappa}\right)+v_{t}^{\kappa}, v_{t}^{\kappa} \sim N\left(0, \sigma_{\kappa}\right),
$$

with $\kappa \in\{i, m, p, b, l, y, w\}$. Finally, we assume that the central bank's target and government expenses on production ratio evolve according to

$$
\begin{aligned}
& \bar{\pi}_{t}=\bar{\pi}^{1-\rho_{\pi}} \bar{\pi}_{t-1}^{\rho_{\pi}} \varepsilon_{t}^{p} \\
& \ln \left(\varepsilon_{t}^{g}\right)=\left(1-\rho_{g}\right) \ln \left(\bar{g}_{y}\right)+\rho_{g} \ln \left(\varepsilon_{t-1}^{g}\right)+v_{t}^{g}, \\
& v_{t}^{g} \sim N\left(0, \sigma_{g}\right) .
\end{aligned}
$$

Then, before starting estimation procedures, we need to make the model stationary. Indeed, as the model features a balanced growth trend, it is necessary to turn it into its intensive form for simulations. All real variables of interest are deflated 
by the deterministic trend $(1+g)^{t}$. We then rewrite the model's equations with intensive variables. ${ }^{5}$

\section{Priors Distributions, Calibration, and Data}

We use Bayesian techniques to estimate the main free parameters of the model. Broadly speaking, we compute by numerical simulation the maximum of the posterior density of the parameters by confronting a priori knowledge about them, through the likelihood function, against data. ${ }^{6}$ The first column of Table 1 shows the different priors set to estimate both the U.S. and euro-area models.

Almost all of the model's parameters are estimated, with the following exceptions: The time preference parameter $\beta$ is set at 0.998 ; the Kimball function's parameter $\zeta_{Y}$ and $\zeta_{L}$ is calibrated at 1.02 as in Dotsey and King (2005); and the average quarterly growth rate of gross domestic product (GDP), $g$, is set at 0.66 percent for the euro area and 0.37 percent for the U.S. economy, based on our database. Note that the prior density functions are quite noninformative for most of the estimated parameters except for inertia coefficients of productivity shocks and what we call "government shocks." We used the previous result of highly persistent shocks in previous works as a prior belief (e.g., Smets and Wouters, 2007).

The data sources are as follows. We use time series from 1970:Q1 to 2007:Q4 (United States) and 2006:Q4 (euro area). For U.S. data, the GDP, consumption, investment, and GDP deflator are from the Bureau of Economic Analysis national accounts. The capacity utilization rate and nominal interest rate- the federal funds effective rateare from the Federal Reserve Board database. For the euro-area data, GDP, consumption, investment, short-term interest rate, and GDP deflator are from the Area-wide Model (AWM) database (Fagan, Henry, and Mestre, 2001). Capacity utilization rate data are from the Eurostat database. Finally, data on labor markets have been used to

\footnotetext{
5 As written in the technical appendix.

6 See Schorfheide (2000) and Smets and Wouters (2003).
}

detrend extensive variables. Total U.S. employment and hours worked for the U.S. and euroarea economies are from the OECD's Economic Outlook database (OECD, 2005). European total employment data are from the AWM database. All extensive variables, namely GDP, consumption, and investment, are first detrended through a Hodrick-Prescott (HP) filter with parameter 1600 using a trend in labor that consists of total hours worked. Then, these variables are deflated by the GDP deflator. We therefore compute the average quarterly growth rate of real gross productivity and detrend again all extensive variables by the corresponding deterministic time trend. Finally, these variables are divided by the mean of GDP over the period. ${ }^{7}$

\section{Implementing the Production Function Method}

The first step consists of estimating the benchmark DSGE model for the two economies and checking the consistency of estimates given by the two last columns of Table $1 .^{8}$ We then simulate the model to obtain consistent time series for production, investment, labor, and capacity utilization.

We now are able to (i) compute the physical capital stock series according to the permanent inventory method (PIM) and, taking into account the deterministic trend,

$$
k_{t}^{P I M}=\frac{1-\bar{\delta}}{1+g} k_{t-1}^{P I M}+i_{t},
$$

as well as the age of capital,

$$
\operatorname{age}_{t}=\frac{1-\bar{\delta}}{1+g} \frac{k_{t-1}^{P I M}}{k_{t}^{P I M}}\left(\text { age }_{t-1}+1\right), \text { and }
$$

(ii) extract the Solow residual, $s_{t}$, as

$$
s_{t}=\ln \left(y_{t}\right)-\alpha \ln \left(k_{t}^{P I M}\right)-(1-\alpha) \ln (L) .
$$

\footnotetext{
7 We deliberately exclude data on wages and labor in the estimation process primarily because of the lack of labor market sophistication in the model.

8 As a consistency check, one can verify that the posterior modes obtained by the estimation process correspond to the maximum of the likelihood function in the parameter direction. Such representations are given in the technical appendix.
} 


\section{Table 1}

\section{Priors Distributions and Posterior Modes}

\begin{tabular}{|c|c|c|c|c|c|}
\hline \multirow[b]{2}{*}{ Parameter } & \multicolumn{3}{|c|}{ Prior distribution } & \multicolumn{2}{|c|}{ Posterior modes } \\
\hline & Type & Mean & SD & Euro area & United States \\
\hline \multicolumn{6}{|c|}{ Preferences } \\
\hline$\theta$ & beta & 0.500 & 0.2000 & 0.3210 & 0.2248 \\
\hline$\sigma_{c}$ & norm & 1.500 & 0.5000 & 1.1592 & 1.4674 \\
\hline$\sigma_{l}$ & norm & 2.000 & 0.5000 & 1.9061 & 0.6218 \\
\hline \multicolumn{6}{|c|}{ Production and technology } \\
\hline$d$ & gamma & 1.500 & 0.2000 & 1.6581 & 1.8098 \\
\hline $\bar{\delta}$ & beta & 0.500 & 0.2000 & 0.0820 & 0.0569 \\
\hline$\phi$ & norm & 5.500 & 5.0000 & 0.1824 & 0.0890 \\
\hline$\alpha$ & beta & 0.500 & 0.2000 & 0.2409 & 0.1907 \\
\hline \multicolumn{6}{|c|}{ Kimball aggregators } \\
\hline$\omega_{Y}$ & norm & -6.000 & 5.0000 & -5.1063 & -4.4232 \\
\hline$\omega_{L}$ & norm & -18.000 & 5.0000 & -16.0926 & -16.1249 \\
\hline \multicolumn{6}{|c|}{ Calvo settings } \\
\hline$\xi_{p}$ & beta & 0.500 & 0.2000 & 0.5411 & 0.4252 \\
\hline$\gamma_{p}$ & beta & 0.500 & 0.2000 & 0.0432 & 0.1295 \\
\hline$\xi_{w}$ & beta & 0.500 & 0.2000 & 0.4431 & 0.5100 \\
\hline$\gamma_{w}$ & beta & 0.500 & 0.2000 & 0.7980 & 0.7781 \\
\hline \multicolumn{6}{|c|}{ Steady-state values } \\
\hline $\bar{z}$ & norm & 0.814 & 0.1000 & 0.8255 & 0.8040 \\
\hline $\bar{g}_{y}$ & beta & 0.200 & 0.1000 & 0.1935 & 0.0461 \\
\hline $\bar{\pi}$ & norm & 1.014 & 0.1000 & 1.0077 & 1.0125 \\
\hline $\bar{L}$ & norm & 1.000 & 0.1000 & 1.0002 & 1.0001 \\
\hline $\bar{y}$ & norm & 1.000 & 0.1000 & 0.8602 & 0.9178 \\
\hline \multicolumn{6}{|c|}{ Autoregressive parameter of shocks } \\
\hline$\rho_{a}$ & beta & 0.990 & 0.0010 & 0.9908 & 0.9904 \\
\hline$\rho_{i}$ & beta & 0.500 & 0.2000 & 0.1739 & 0.1655 \\
\hline$\rho_{\pi}$ & beta & 0.500 & 0.2000 & 0.0510 & 0.0961 \\
\hline$\rho_{m}$ & beta & 0.500 & 0.2000 & 0.9686 & 0.9391 \\
\hline$\rho_{p}$ & beta & 0.500 & 0.2000 & 0.0510 & 0.0960 \\
\hline$\rho_{l}$ & beta & 0.500 & 0.2000 & 0.4999 & 0.4989 \\
\hline$\rho_{g}$ & beta & 0.970 & 0.0100 & 0.9750 & 0.9980 \\
\hline$\rho_{b}$ & beta & 0.500 & 0.2000 & 0.9662 & 0.9571 \\
\hline$\rho_{w}$ & beta & 0.500 & 0.2000 & 0.5007 & 0.4998 \\
\hline$\rho_{y}$ & beta & 0.900 & 0.0500 & 0.8473 & 0.9180 \\
\hline
\end{tabular}




\section{Table 1, cont'd}

\section{Priors Distributions and Posterior Modes}

\begin{tabular}{|c|c|c|c|c|c|}
\hline \multirow[b]{2}{*}{ Parameter } & \multicolumn{3}{|c|}{ Prior distribution } & \multicolumn{2}{|c|}{ Posterior modes } \\
\hline & Type & Mean & SD & Euro area & United States \\
\hline \multicolumn{6}{|l|}{ Taylor rule } \\
\hline$\phi_{\pi}$ & norm & 2.000 & 0.5000 & 2.5860 & 2.6345 \\
\hline$\phi_{y}$ & norm & 0.100 & 0.0500 & 0.0905 & 0.1394 \\
\hline$r_{\Delta y}$ & norm & 0.000 & 0.5000 & 0.3419 & 0.6927 \\
\hline$r_{\Delta \pi}$ & norm & 0.300 & 0.1000 & 0.1384 & 0.1252 \\
\hline$\rho_{r}$ & beta & 0.500 & 0.2000 & 0.9268 & 0.8505 \\
\hline \multicolumn{6}{|c|}{ Standard deviation of shocks } \\
\hline$v_{i}$ & invg & 0.100 & 2.0000 & 0.0208 & 0.0512 \\
\hline$v_{a}$ & invg & 0.010 & 2.0000 & 0.0072 & 0.0061 \\
\hline$v_{p}$ & invg & 0.001 & 2.0000 & 0.0033 & 0.0026 \\
\hline$v_{m}$ & invg & 0.001 & 2.0000 & 0.0004 & 0.0006 \\
\hline$v_{b}$ & invg & 0.100 & 2.0000 & 0.0018 & 0.0020 \\
\hline$v_{l}$ & invg & 0.001 & 2.0000 & 0.0005 & 0.0005 \\
\hline$v_{g}$ & invg & 0.001 & 2.0000 & 0.0197 & 0.0786 \\
\hline$v_{w}$ & invg & 0.001 & 2.0000 & 0.0005 & 0.0005 \\
\hline$v_{y}$ & invg & 0.100 & 2.0000 & 0.0184 & 0.0209 \\
\hline Data density & & & & 3,634 & 3,598 \\
\hline
\end{tabular}

NOTE: This table shows prior distribution of the benchmark model parameters and estimation results at the mode of the marginal density posteriors. Prior probability density functions are normal (norm), beta (beta), or inverse gamma (invg). SD, standard deviation.

\section{Table 2}

\section{Results Estimates of TFP Equation}

\begin{tabular}{|c|c|c|c|c|c|}
\hline Study area & $\begin{array}{c}\gamma_{0} \\
\text { intercept }\end{array}$ & $\begin{array}{c}\gamma_{1} \\
s_{t-1}\end{array}$ & $\begin{array}{c}\gamma_{2} \\
\ln \left(z_{t}\right)\end{array}$ & $\begin{array}{c}\gamma_{3} \\
\text { age }_{t}\end{array}$ & $R^{2}$ \\
\hline Euro area & $\begin{array}{c}-0.0100 \\
(0.0061)\end{array}$ & $\begin{array}{c}0.9059 \\
(0.0081)\end{array}$ & $\begin{array}{c}-0.1226 \\
(0.0123)\end{array}$ & $\begin{array}{c}-4.3 \mathrm{e}-03 \\
(7.0 \mathrm{e}-04)\end{array}$ & $\begin{array}{c}0.9974 \\
-\end{array}$ \\
\hline United States & $\begin{array}{c}-0.0300 \\
(0.0082)\end{array}$ & $\begin{array}{c}0.8784 \\
(0.0090)\end{array}$ & $\begin{array}{c}-0.1477 \\
(0.0123)\end{array}$ & $\begin{array}{c}-2.2 \mathrm{e}-03 \\
(5.8 \mathrm{e}-04)\end{array}$ & $\begin{array}{c}0.9946 \\
-\end{array}$ \\
\hline
\end{tabular}

NOTE: This table shows results estimates of the TFP equation based on simulated series of 3,000 occurrences, where the first 1,000 have been dropped. We made 1,000 regressions. The figures in the table correspond to the average parameters over these regressions. Average standard deviations are listed in parentheses. 


\section{Figure 1}

\section{Impulse Response Function for Production and DSGE/PFA Measures of Potential Output (United States)}
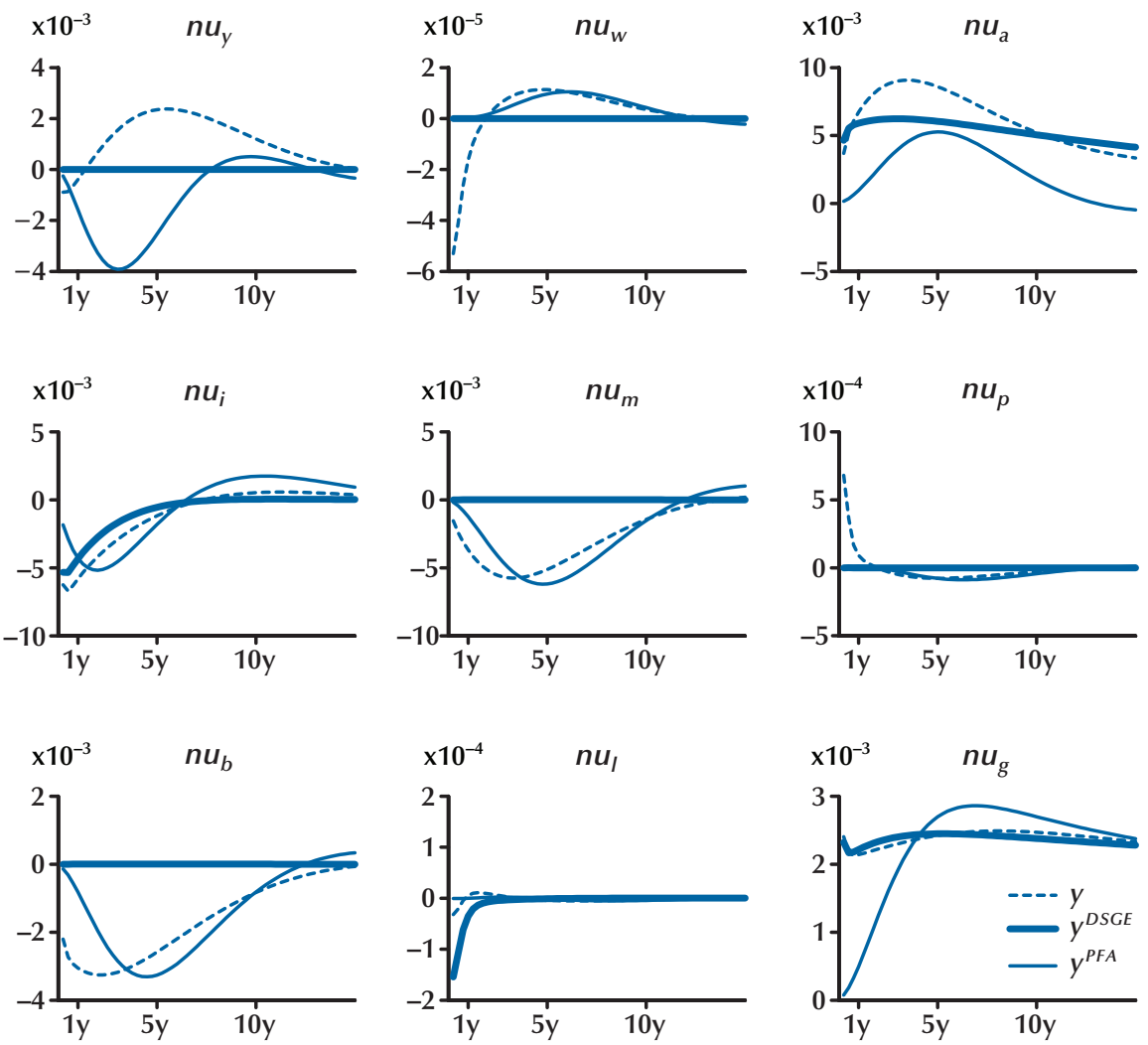

Finally, we estimate the following TFP equation:

$$
s_{t}=\gamma_{0}+\gamma_{1} s_{t-1}+\gamma_{2} \ln \left(z_{t}\right)+\gamma_{3} a g e_{t}+\varepsilon_{t},
$$

where $\varepsilon_{t}$ is an i.i.d. process. ${ }^{9}$

Table 2 gives the estimates of the TFP equation for both the U.S. and euro-area model economies. It is worth noting that results show a negative coefficient on the capacity utilization, contrary to what we assumed as an economic intuition in the section on benchmarking the DSGE model.

We then compute the potential production based on the PFA. First, we assume that potential capital is taken as $k_{t}^{P I M}$, as computed from the PIM. Then, we use filtered data to assess poten-

9 See Cahn and Saint-Guilhem (2009). tial employment, $L_{t}^{\text {Filt }}{ }^{10}$ Finally, we define the medium-term potential TFP, $\hat{s}_{t}^{M T}$, from our previous estimates by setting $z_{t} \equiv \bar{Z}$ and eliminating the lagged term ${ }^{11}$ :

${ }^{10}$ More specifically, we use a moving average version of the HP filterformally, if a process, $x_{t}$, can be split between a cyclical part, $c_{t}$, and a smooth trend, $m_{t}$. The HP filter defines the cyclical part as

$$
c_{t}=\frac{\lambda(1-\mathfrak{R})^{2}\left(1-\mathfrak{L}^{-1}\right)^{2}}{1+\lambda(1-\mathfrak{Z})^{2}\left(1-\mathfrak{L}^{-1}\right)^{2}} x_{t},
$$

where $\mathbb{R}$ is the lag operator. Expanding this expression and considering that $c_{t}=x_{t}-m_{t}$, we use the following relation to define potential labor:

$$
L_{t}=L_{t}^{F i l t}+\lambda\left(\mathfrak{L}^{-2}-4 \mathfrak{Q}^{-1}+6 I-4 \mathfrak{Q}+\mathfrak{R}^{2}\right) L_{t}^{F i l t} .
$$

Finally, we set $\lambda=1600$ as is standard for quarterly economic time series.

${ }^{11}$ See Cahn and Saint-Guilhem (2009). 


\section{Figure 2}

\section{Impulse Response Function for Production and DSGE/PFA Measures of Potential Output (Euro Area)}
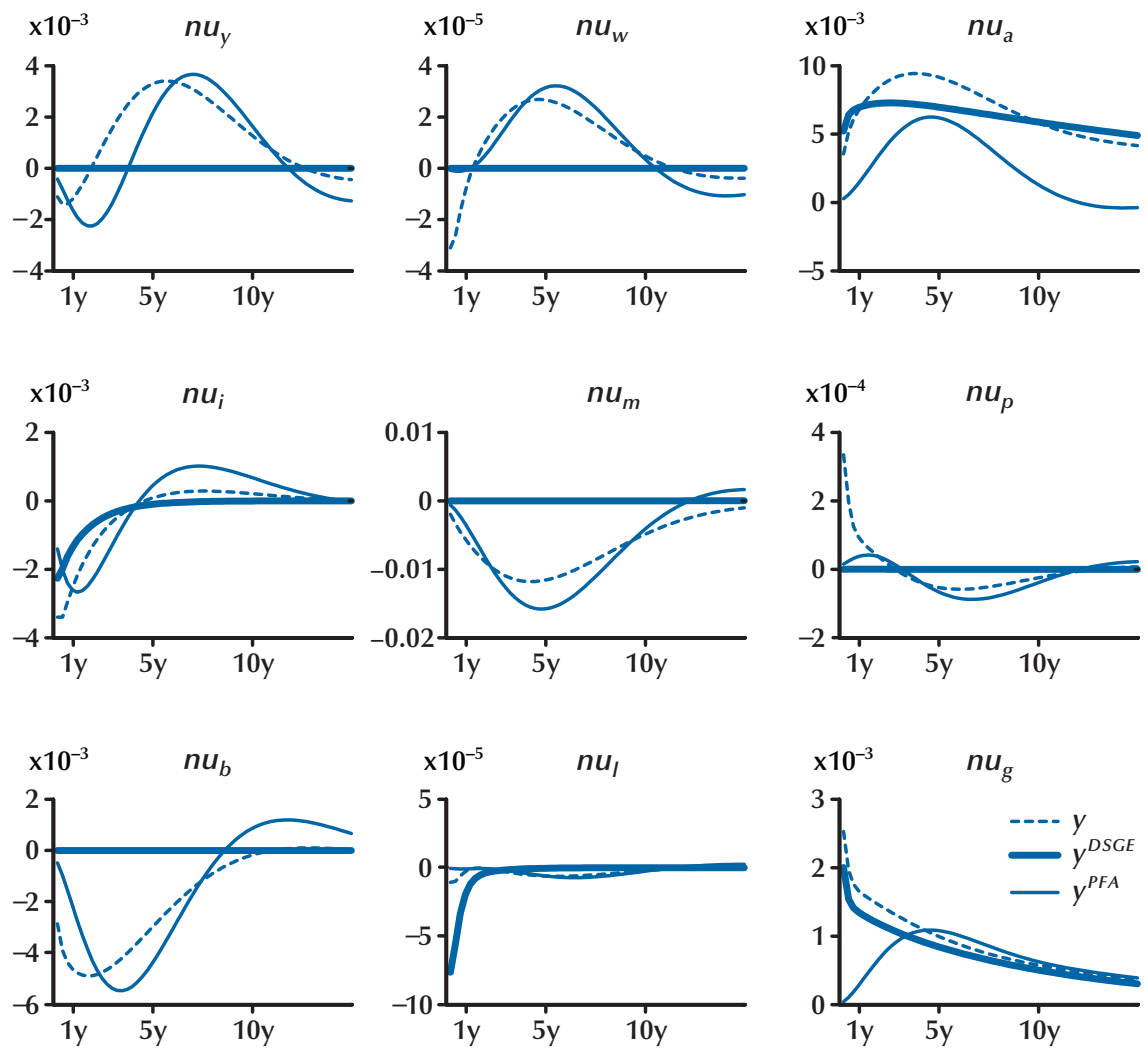

$$
\hat{s}_{t}^{M T}=\frac{\gamma_{0}}{1-\gamma_{1}}+\frac{\gamma_{2}}{1-\gamma_{1}} \ln (\bar{z})+\frac{\gamma_{0}}{1-\gamma_{1}} \text { age } e_{t+1} .
$$

Consequently, the potential output based on our production function method is given by

$$
y_{t}^{P F A}=e^{\hat{s}_{t}^{M T}}\left(k_{t}^{P I M}\right)^{\alpha}\left(L_{t}^{F i l t}\right)^{1-\alpha} .
$$

\section{DISCUSSION}

In this section, we analyze and compare the dynamic behavior of the DSGE and PFA estimates of potential output through impulse response functions (IRFs) and variance decomposition. In the following, the terms "U.S. economy/model" and "euro-area economy/model" refer to the models estimated on U.S. data or euro-area data, respectively.

\section{IRF Analysis}

Figures 1 and 2 plot the IRFs of the stochastic shocks for actual output and both DGSE- and PFA-based measures of potential output, calculated with the estimated parameters given in Table 1. Figures 3 through 8 show the IRFs for various factors (output, consumption, investment, nominal interest rate, inflation, and DSGE- and PFA-based output gaps). Results for U.S. and euro-area models are broadly similar, except for the response to an external expenses shock, $v^{g}$, for which the U.S. response appears to be much more inert than the euro-area responses. The fol- 
Figure 3

Impulse Response Function for Output, Consumption, and Investment (United States)
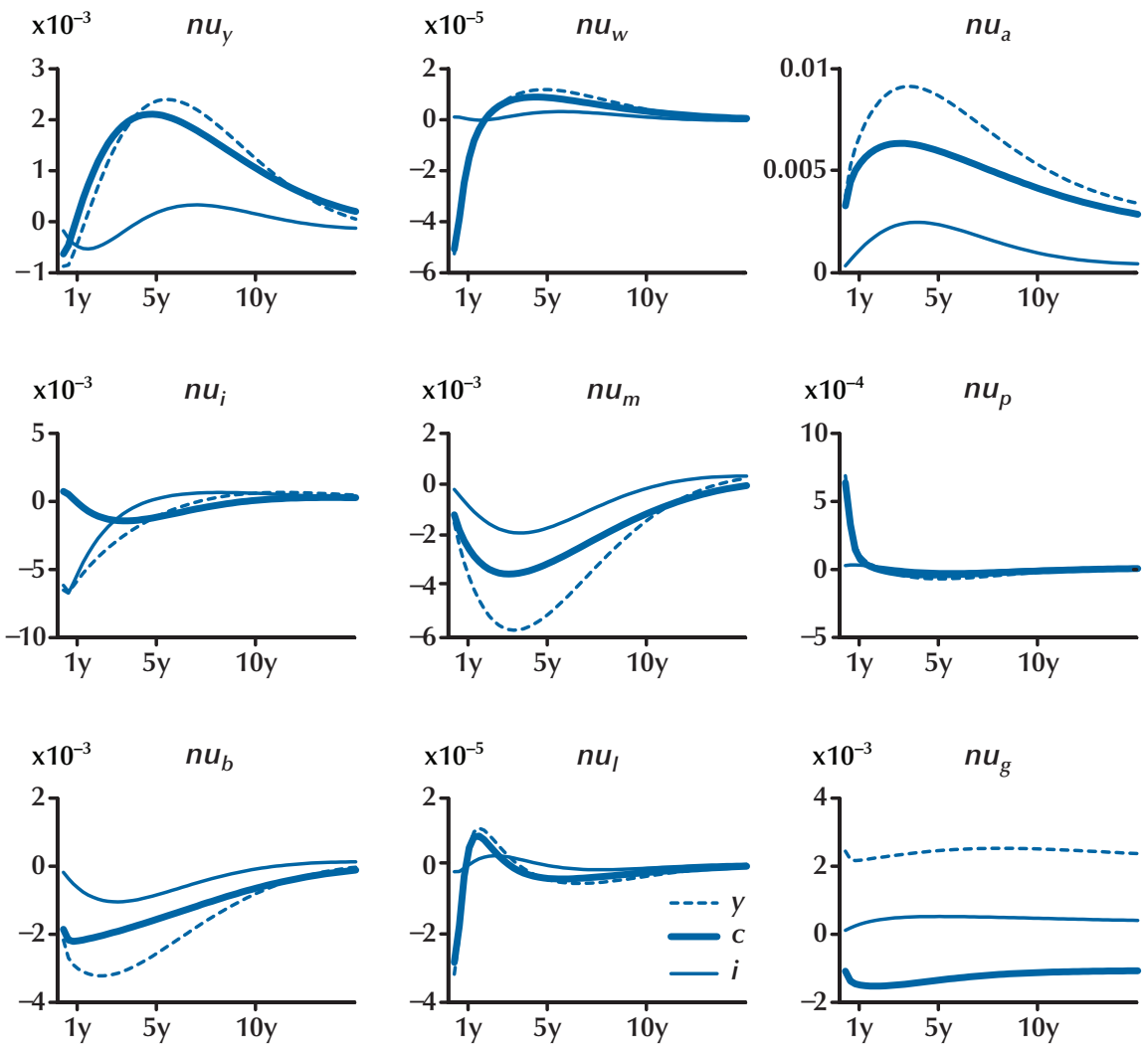

lowing analysis applies for both economies, apart from this shock.

The figures show that after a positive productivity shock, $v^{a}$, actual, DSGE, and PFA potential outputs rise together, but the PFA measure rises more gradually. Moreover, the response of the model-based potential output seems to be more persistent for the DSGE than for the PFA. Indeed, a positive productivity shock results in an increase in investment, and therefore the age of capital stock grows gradually, as do the medium-term TFP and PFA potential outputs. On the other hand, the productivity shock instantly affects both the productivity term and the Solow residual. Consequently, after such a shock, both actual and PFA potential outputs evolve similarly, but the gap between them remains constant for a longer time than with the DSGE potential output (see Figures 5 and 8).

The effect of a positive-quantitatively negative in its effect-investment shock, $v^{i}$, leads to similar dynamics for the three output measures. The shock deforms the adjustment cost function, leading to an increase in the cost of new capital. Hence, investment falls and capital stock shows a hump-shaped decrease, reflected in its age and then in potential TFP. Interestingly, all these variables cross their steady-state path simultaneously after about 6 years. Before, the PFA potential output is below the DSGE potential output, and this order changes after the date; the actual output lies between the two measures. This implies that the two related gap measures evolve in opposite directions after an investment shock. 


\section{Figure 4}

\section{Impulse Response Function for Nominal Interest Rate and Inflation (United States)}
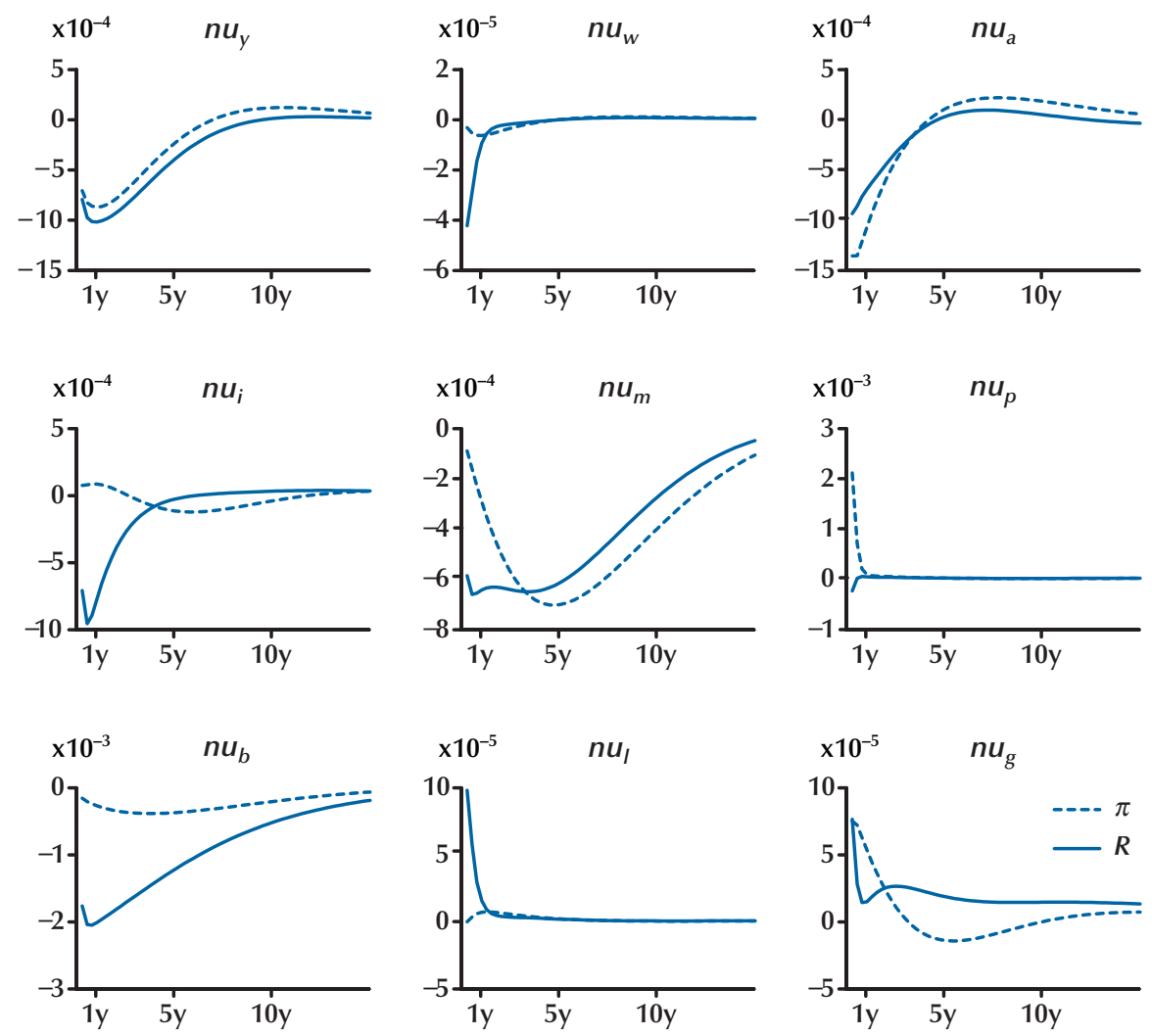

With respect to a positive labor supply shock, $v^{l}$, PFA potential output does not react, whereas DSGE potential output decreases instantaneously, as does actual output but to a lesser extent. In fact, labor in the world without nominal rigidities can adjust more rapidly, and the reaction of DSGE potential output is one order of magnitude higher than for actual and PFA potential outputs.

Conversely, after a positive government shock, $v^{g}$, actual and DSGE potential outputs shift upward suddenly, whereas PFA potential output gradually reaches their level. After the shock, demand for output shifts upward instantly, coinciding with a higher level of employment. Hence, potential employment grows gradually and then results in the slower increase in PFA-based potential output. Note that the response to the govern- ment shock of the U.S. model is more persistent than for the euro-area ones. This is mainly due to a more persistent stochastic structure of the shock estimated for the United States.

Not surprisingly, DSGE potential output does not respond to any nominal shocks-namely, markup, monetary, or equity premium shocks—as these shocks do not enter into the real side model. The most remarkable fact is that PFA potential output reacts significantly to such shocks. After a positive monetary shock to the interest rate, $v^{m}$, both actual and PFA output show a hump-shaped decrease. The qualitative effects of the equity premium shock, $v^{b}$, are quite similar.

The model economies show similar responses to the price and wage markup shocks with first an instantaneous fall in actual output and then a 
Figure 5

Impulse Response Function for Inflation-, DSGE-, and PFA-Based Output Gaps (United States)
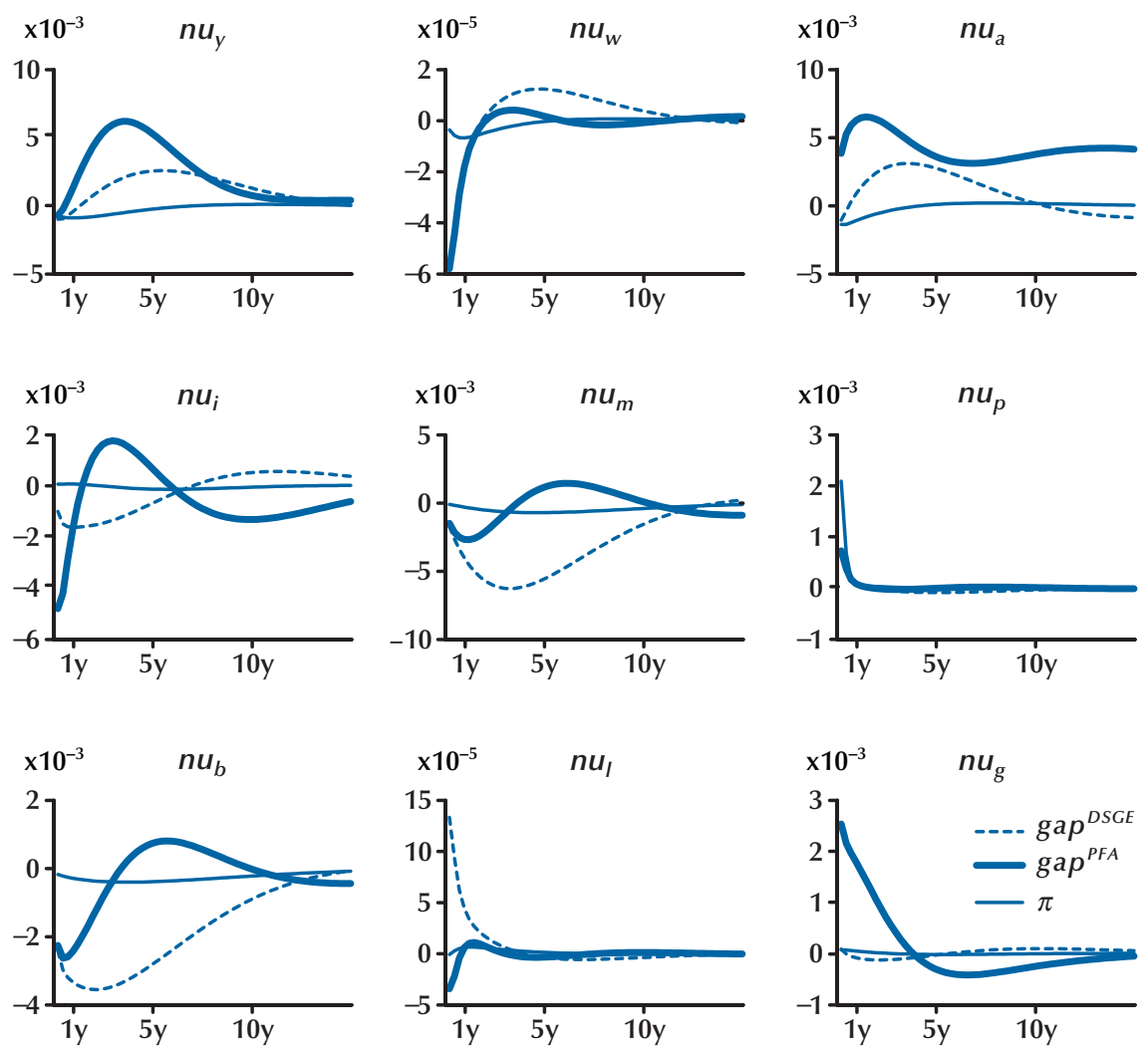

hump-shaped increase. Nevertheless, the actual output reaction to a wage markup shock is about two orders of magnitude less than the response to a price markup shock. PFA potential output responds to these shocks in a similar manner but more gradually, generating a persistent drift.

\section{Variance Decomposition}

Table 3 shows the contribution of each structural shock to the asymptotic forecast error variance of the endogenous variables shown in Table 4. For the U.S. economy, the productivity shock seems to dominate asymptotically the sources of actual and DSGE-based potential outputs by about 50 percent and 60 percent, respectively. A government spending shock is the other main source of fluctuations, accounting for 27 percent of actual production and 37 percent of DSGE potential.

The interest rate shock appears to create the most striking difference between actual and DSGE potential output variance-it amounts to about 55 percent of the related output gap measure. For the PFA potential measure, the external spending shock accounts for 43 percent of the variance as the main contributor. The productivity shock contribution reaches only 15 percent, less than the interest shock (21 percent). All in all, contrary to the DSGE-based measure, the productivity shock accounts for 68 percent of the PFA output gap variance.

For the euro-area model economy, the variance decomposition of actual production is quite 


\section{Figure 6}

\section{Impulse Response Function for Output, Consumption, and Investment (Euro Area)}
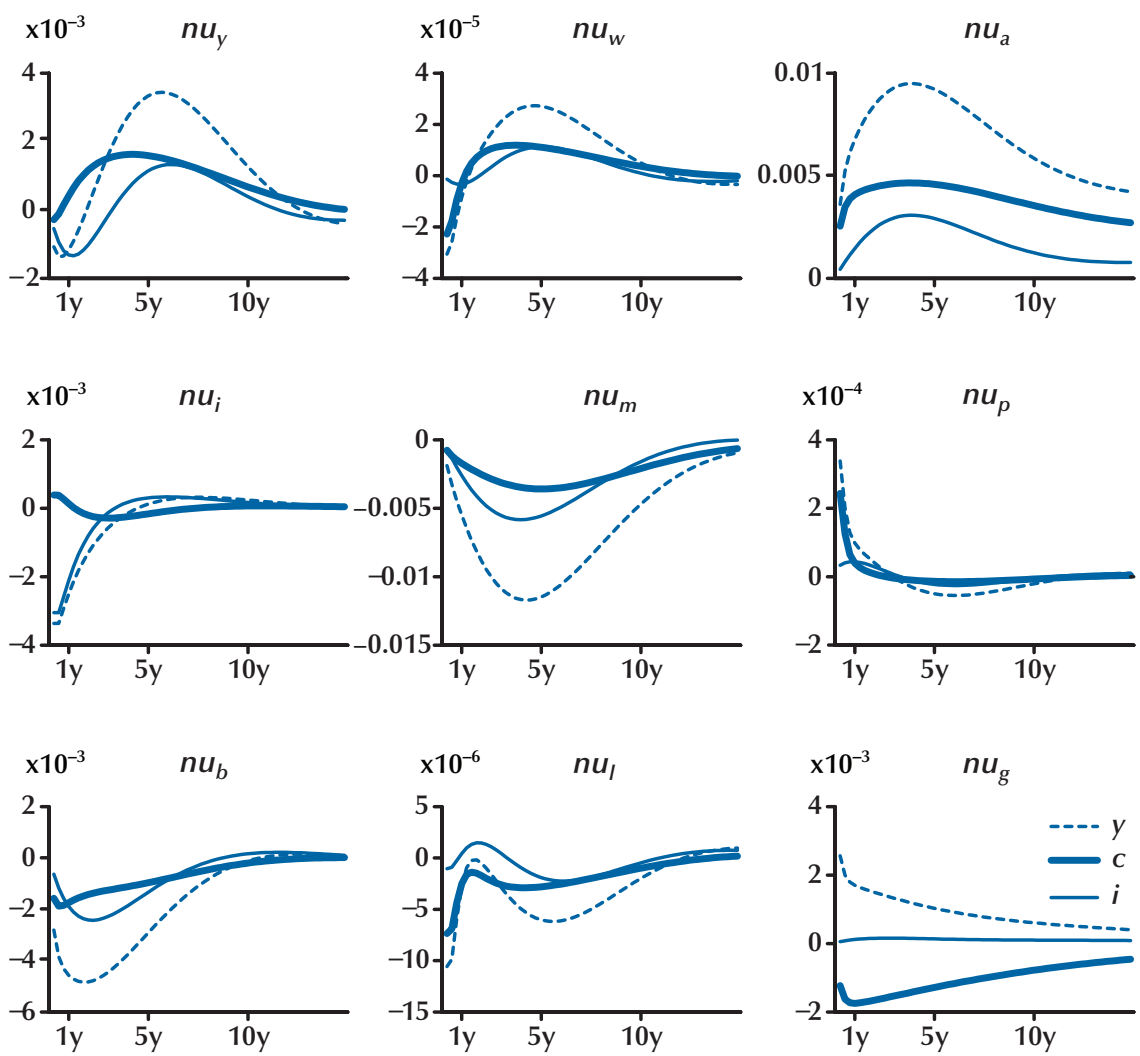

similar to that of the United States. Nevertheless, almost all the variance of DSGE-based potential output seems to be derived from the productivity shock, whereas the largest part of the PFA potential output variance comes from the interest shock (76 percent) and productivity shock to a lesser extent (12 percent). As a result, DSGE output gap variations come primarily from the interest shock (82 percent), and PFA gap variance is derived from the productivity shock (74 percent).

Finally, Table 4 shows that both DSGE and PFA potential growth are less volatile than actual output. Nevertheless, one could not conclude that the PFA-based measure is smoother than the DSGE one.

\section{Implications}

Our analysis suggests that the PFA and the DSGE approaches to potential output measurement differ significantly, at least from a business cycle perspective. For two different modelsone close to the U.S. data, the other to the euroarea data-the output gap related to the DSGE measure captures mainly nominal shocks, which in summation amounts to more than 80 percent (about 97 percent for the U.S. model) of the gap variance. Alternatively, the PFA gap reacts mainly to productivity shock (about 70 percent of the variance.)

As a consequence, it seems to us that using the PFA to compute potential output and the related output gap presents some drawbacks related to 
Figure 7

Impulse Response Function for Nominal Interest Rate and Inflation (Euro Area)
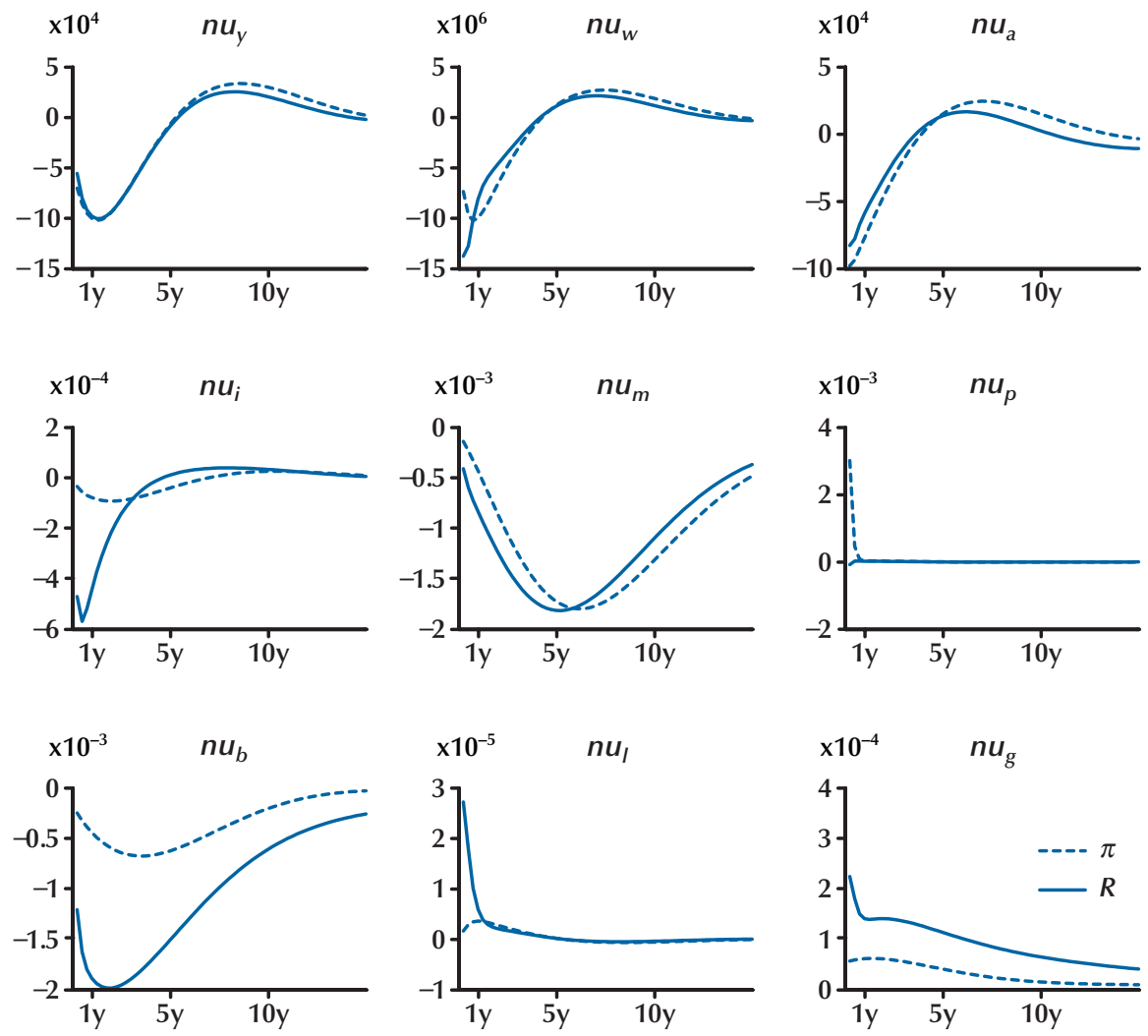

its ability to properly reflect inflationary pressures related to nominal shocks. In contrast, the DSGEbased potential output measure could lead to misstatements about potential growth as this measure reacts to temporary but persistent shocks such as productivity shocks. These two assessments can lead to contradictions in terms of economic diagnostics. For instance, assuming that the model is the one that generates the actual data, one could think that during the 1990-95 period, GDP growth in the United States (2.4 percent) was below its potential based on the DSGE measure (2.7 percent), as stated in Table 5. One would reach an opposing conclusion using the PFA-based measure (1.7 percent). The same contradiction holds for the euro-area economy during the 2000-05 period.
From an empirical point of view, these results tend to moderate the possible structural interpretations of the international comparison based on the PFA. Indeed, if one believes that some structural shocks drive the dynamics of economic variables and wants to compare potential growth of several economies using the PFA, the fact that the results depend on the idiosyncratic shocks faced by each economy must be considered. Consequently, this argues for a normalization of such structural shocks before applying the PFA. For instance, based on the PFA (left side of Table 5), it appears that actual growth in the euro-area economy stood below its PFA potential in the past 15 years. Conversely, the U.S. economy's actual growth was above its PFA potential. Moreover, the U.S. PFA potential was higher than the 


\section{Figure 8}

\section{Impulse Response Function for Inflation-, DSGE-, and PFA-Based Output Gaps (Euro Area)}
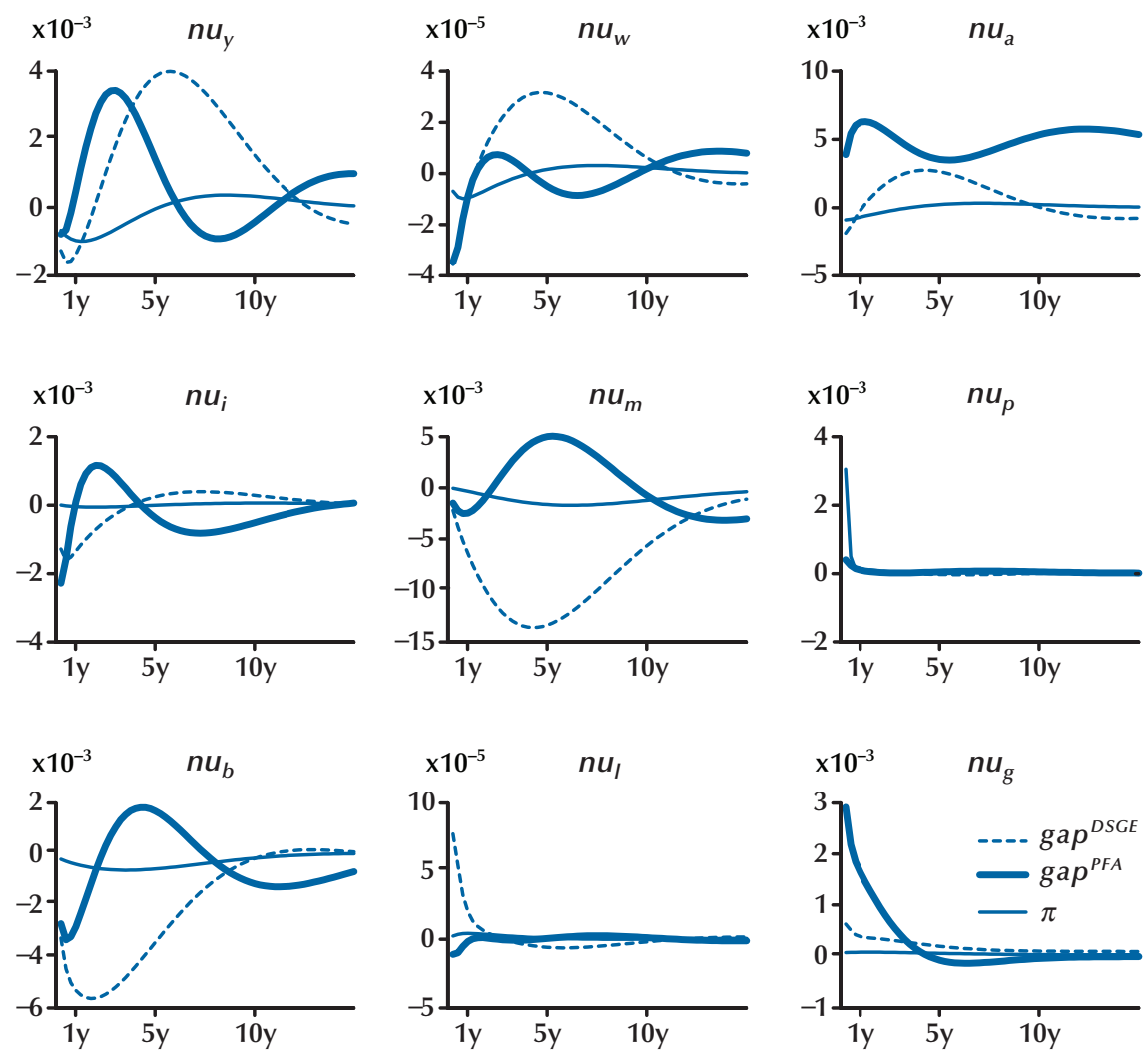

euro area's. Does it clarify the need for structural reforms in the European economy to keep pace with the U.S. economy? Imagine that both economies interchanged the structural shocks they faced. Would we observe identical behavior? The three right columns of Table 5 present the results of such an experiment; they lead to the exact opposite conclusion regarding the comparison between the United States and the euro area.

Alternatively, a monetary authority that must conduct interest rate policy based on a Taylor rule that includes an output gap measure could make the opposite decision depending on the method used to measure drift between actual and potential output. For instance, after a positive productivity shock, the central bank could decide to instantaneously increase the nominal interest rate if based on the PFA gap estimates, whereas the decision would be to decrease the interest rate (as shown in Figure 4), at least in a DSGE framework.

\section{CONCLUSION}

In this article, we compared the PFA measure of potential output with the DSGE definition of potential output in a fully integrated framework. We estimated a DSGE model for U.S. and euroarea data and integrated into the two versions of the model a PFA measure of potential output fully consistent with the model. Results have shown that, in a DSGE framework, the PFA leads to potential output measures that are not exempt from 
Table 3

Variance Decomposition

\begin{tabular}{|c|c|c|c|c|c|c|c|c|c|}
\hline Shocks & $y_{t}$ & $y_{t}^{D S G E}$ & $y_{t}^{P F A}$ & $\pi_{t}$ & $c_{t}$ & $i_{t}$ & $\boldsymbol{R}_{t}$ & $\operatorname{gap}_{t}^{D S G E}$ & $\operatorname{gap}_{t}^{P F A}$ \\
\hline \multicolumn{10}{|l|}{ United States } \\
\hline Productivity shock & 51 & 59 & 15 & 24 & 65 & 28 & 4 & 14 & 67 \\
\hline Inflation target shock & - & - & - & 11 & 11 & - & - & - & - \\
\hline Labor supply shock & - & - & - & - & - & - & - & - & - \\
\hline External spending shock & 27 & 37 & 43 & - & 15 & 8 & - & - & 1 \\
\hline Investment shock & 5 & 4 & 10 & 1 & 1 & 45 & 4 & 3 & 4 \\
\hline Equity premium shock & 4 & - & 6 & 11 & 4 & 4 & 68 & 18 & 2 \\
\hline Interest rate shock & 11 & - & 21 & 32 & 11 & 14 & 12 & 55 & 4 \\
\hline Price distortion shock & 2 & - & 5 & 21 & 4 & 1 & 12 & 10 & 22 \\
\hline Wage distortion shock & - & - & - & - & - & - & - & - & - \\
\hline \multicolumn{10}{|l|}{ Euro area } \\
\hline Productivity shock & 48 & 99 & 12 & 4 & 67 & 22 & 2 & 3 & 73 \\
\hline Inflation target shock & - & - & - & 7 & - & - & - & - & - \\
\hline Labor supply shock & - & - & & - & - & - & - & - & - \\
\hline External spending shock & 1 & 1 & 1 & - & 5 & - & - & - & 1 \\
\hline Investment shock & 1 & - & 1 & - & - & 3 & 1 & - & 1 \\
\hline Equity premium shock & 5 & - & 7 & 8 & 3 & 7 & 44 & 10 & 3 \\
\hline Interest rate shock & 42 & - & 75 & 72 & 22 & 65 & 48 & 82 & 18 \\
\hline Price distortion shock & 3 & - & 4 & 9 & 3 & 3 & 5 & 5 & 4 \\
\hline Wage distortion shock & - & - & - & - & - & - & - & - & - \\
\hline
\end{tabular}

NOTE: This table presents the theoretical variance decomposition among the model's shocks (expressed in percent).

\section{Table 4}

\section{Theoretical Moments}

\begin{tabular}{lcccc} 
& \multicolumn{2}{c}{ United States } & \multicolumn{2}{c}{ Euro area } \\
\cline { 2 - 4 } Variable & Mean & SD & Mean & 0.0909 \\
\hline$y$ & 0.9178 & 0.0794 & 0.8602 & 0.0611 \\
$y^{D S G E}$ & 0.9178 & 0.0667 & 0.8602 & 0.0819 \\
$Y^{P F A}$ & 0.9178 & 0.0620 & 0.8602 & 0.0433 \\
$C$ & 0.7237 & 0.0525 & 0.5083 & 0.0117 \\
$\pi$ & 1.0125 & 0.0066 & 1.0077 & 0.0143 \\
$R$ & 1.0239 & 0.0105 & 1.0136 & 0.0347 \\
gap $^{D S G E}$ & 0.1517 & 0.0229 & 0.1855 & 0.0755 \\
gap $^{P F A}$ & 0.0000 & 0.0394 & 0.0000 & 0.0598 \\
\hline
\end{tabular}




\section{Table 5}

\section{Annual Potential Growth Comparison}

\begin{tabular}{|c|c|c|c|c|c|c|}
\hline \multirow[b]{2}{*}{ Period } & \multicolumn{3}{|c|}{ United States } & \multicolumn{3}{|c|}{ United States* } \\
\hline & $y$ & $y^{D S G E}$ & $y^{P F A}$ & $y$ & $y^{\text {DSGE }}$ & $y^{P F A}$ \\
\hline 1990-1995 & 2.4 & 2.7 & 1.7 & 2.0 & 1.6 & 2.6 \\
\hline 1995-2000 & 3.9 & 3.4 & 3.8 & 2.4 & 2.5 & 3.0 \\
\hline 2000-2005 & 2.4 & 1.9 & 1.7 & 0.0 & 1.2 & 0.6 \\
\hline \multirow[t]{3}{*}{ 1990-2005 } & 2.9 & 2.6 & 2.4 & 1.5 & 1.8 & 2.1 \\
\hline & \multicolumn{3}{|c|}{ Euro area } & \multicolumn{3}{|c|}{ Euro area $^{\dagger}$} \\
\hline & $y$ & $y^{D S G E}$ & $y^{P F A}$ & $y$ & $y^{\text {DSGE }}$ & $y^{P F A}$ \\
\hline 1990-1995 & 1.8 & 2.5 & 1.5 & 2.4 & 3.6 & 2.2 \\
\hline 1995-2000 & 2.4 & 2.8 & 2.7 & 4.2 & 4.1 & 2.3 \\
\hline 2000-2005 & 1.9 & 1.9 & 2.9 & 4.8 & 3.9 & 3.1 \\
\hline 1990-2005 & 2.0 & 2.4 & 2.4 & 3.8 & 3.8 & 2.5 \\
\hline
\end{tabular}

NOTE: This table shows actual and potential growth on average over different subperiods. Figures are given in percent. They also include both the deterministic and labor trends. *U.S. model simulated with euro-area smoothed shocks. ${ }^{+}$Euro-area model simulated with U.S. smoothed shocks.

the effects of nominal or temporary shocks. The empirical implication of these results is that estimates of potential output based on an ad hoc PFA could be highly dependent on transitory phenomena. Moreover, cross-country differences in potential output based on the PFA are likely to reflect not only structural differences, but also different patterns of shocks across time. This leads to the assessment of the quantitative role of shocks in cross-country differences in potential output.

One way to address this issue is to implement in a DSGE model a scenario comparing potential output across economies confronted by the same shocks across time, while exhibiting differences in structural parameters.

However, to answer this question in a more satisfactory manner, we need to improve the present study in several directions. First, it would be of particular interest to identify the causes of divergences between PFA and DSGE potential output measures. Such an analysis could be conducted parameter by parameter to assess their weight on the discrepancy between the two assessments. Second, one would need to improve the estimation procedure by identifying the marginal posterior density of the model through Markovchain Monte Carlo simulations on the one hand, and by allowing structural breaks in the TFP regression equation on the other hand. Finally, one could study the implications for monetary policy of the use of PFA rather than DSGE measures of output gap in a class of central bank decision rules. Obviously, these studies should be performed with an enhanced model, especially with regard to the modeling of the labor market, with an extension of the model introducing unemployment and participation considerations to account for additional sources of fluctuations in potential output and the output gap.

\section{REFERENCES}

Cahn, Christophe and Saint-Guilhem, Arthur. "Potential Output Growth in Several Industrialised Countries: A Comparison.” Empirical Economics, 2009 (forthcoming).

Calvo, Guillermo A. "Staggered Prices in a UtilityMaximizing Framework." Journal of Monetary Economics, September 1983, 12(3), pp. 383-98. 


\section{Cahn and Saint-Guilhem}

Dotsey, Michael and King, Robert G. "Implications of State-Dependent Pricing for Dynamic Macroeconomic Models." Journal of Monetary Economics, January 2005, 52(1), pp. 213-42.

Edge, Rochelle M.; Kiley, Michael T. and Laforte, Jean-Philippe. "Natural Rate Measures in an Estimated DSGE Model of the U.S. Economy." Finance and Economics Discussion Series No. 2007-08, Federal Reserve Board, Washington, DC; www.federalreserve.gov/pubs/feds/2007/200708/ 200708pap.pdf.

Fagan, Gabriel; Henry, Jerome and Mestre, Ricardo. "An Area Wide Model (AWM) for the Euro Area." ECB Working Paper No. 42, European Central Bank, January 2001;

www.ecb.int/pub/pdf/scpwps/ecbwp042.pdf.

Greenwood, Jeremy; Hercowitz, Zvi and Huffman, Gregory W. "Investment, Capacity Utilization, and the Real Business Cycle." American Economic Review, June 1988, 78(3), pp. 402-17.

International Monetary Fund. "France-2007 Article IV Consultation Concluding Statement." International Monetary Fund, November 19, 2007; www.imf.org/external/np/ms/2007/111907.htm.
Kimball, Miles. "The Quantitative Analytics of the Basic Neomonetarist Model." Journal of Money, Credit, and Banking, 1995, 27(4 Part 2), pp. 1241-77.

Neiss, Katherine and Nelson, Edward. "Inflation Dynamics, Marginal Costs and the Output Gap: Evidence from Three Countries." Journal of Money, Credit, and Banking, December 2005, 37(6), pp. 1019-45.

Organisation for Economic Co-operation and Development. OECD Economic Outlook No. 78. December 2005.

Schorfheide, Frank. "Loss Function-Based Evaluation of DSGE Models.” Journal of Applied Econometrics, 15(6), pp. 645-70.

Smets, Frank and Wouters, Rafael. "An Estimated Dynamic Stochastic General Equilibrium Model of the Euro Area." Journal of the European Economic Association, 2003, 1(5), pp. 1123-75.

Smets, Frank and Wouters, Rafael. "Shocks and Frictions in U.S. Business Cycles: A Bayesian DSGE Approach." American Economic Review, June 2007, 97(3), pp. 586-606. 